\title{
Ionic Liquid Based Electrochemical Ethylene Sensor for Fruit and Vegetable Monitoring
}

\author{
Wout Knoben $^{1}$, Marcel A.G. Zevenbergen ${ }^{1}$, Sywert H. Brongersma ${ }^{1}$, Mercedes Crego-Calama ${ }^{1}$ \\ Holst Centre/imec, High Tech Campus 31, 5656 AE Eindhoven, The Netherlands, \\ wout.knoben@imec-nl.nl
}

\begin{abstract}
:
Ethylene is a plant hormone and an indicator for the ripeness of various fruits and vegetables. Measuring the ethylene concentration is therefore a powerful method for monitoring and controlling the ripening process. However, current ethylene detection equipment is bulky and expensive or lacking sufficient sensitivity and selectivity. At Holst Centre, a novel electrochemical ethylene sensor has been developed, in which a thin ionic liquid film is used as the electrolyte. This enables the development of a small, low power and low cost ethylene sensor. Currently, the detection limit of the sensor is less than $1 \mathrm{ppm}$. This is close to the specification needed for monitoring the ripening process during transport and storage of fruits and vegetables. Moreover, fabrication of the sensor on a flexible substrate is demonstrated. This opens up possibilities for integrating the sensor in packaging materials, so-called smart packaging. In this contribution, the sensor technology will be explained and recent developments will be presented.
\end{abstract}

Key words: ethylene, electrochemical sensor, ionic liquid, ripening, monitoring

\section{Introduction}

Ethylene $\left(\mathrm{C}_{2} \mathrm{H}_{4}\right)$ is a gaseous plant hormone which plays an important role in biological processes such as the shedding of leaves, opening of flowers and ripening of fruits [1]. Measuring the ethylene concentration is therefore a powerful method for monitoring and controlling these processes. This is of great importance in horticulture and during transport and storage of plants, fruits and vegetables. However, ethylene concentrations are not measured routinely in these environments due to a lack of affordable and sufficiently sensitive and selective ethylene detection equipment. Electrochemical sensors could fulfill these requirements and are used successfully for the detection of various gases. However, electrochemical detection of ethylene requires a strongly acidic electrolyte and/or elevated temperature, which are incompatible with food monitoring applications [2].

The sensor technology presented here overcomes the aforementioned drawbacks and allows room temperature detection of ethylene with a miniaturized thin film electrochemical sensor [3]. This is achieved by replacing the acidic aqueous electrolyte by an ionic liquid (IL). lonic liquids (fluids which consist solely of ions at room temperature) have several important advantages over traditional aqueous electrolytes, such as a high ethylene solubility and a negligible vapor pressure [4]. The latter allows the deposition of stable thin ionic liquid films, which leads to high sensitivities and short response times. Moreover, the absence of a large liquid reservoir allows sensor fabrication by standard cleanroom techniques and even on flexible plastic substrates, thus reducing fabrication costs.

The working mechanism of our sensor is schematically shown in figure 1. Ethylene dissolves in a thin ionic liquid film covering a gold working electrode. When a sufficiently high potential is applied, ethylene oxidation takes place and electrons are transferred to the electrode. The resulting current is proportional to the ethylene concentration, which can thus be determined.

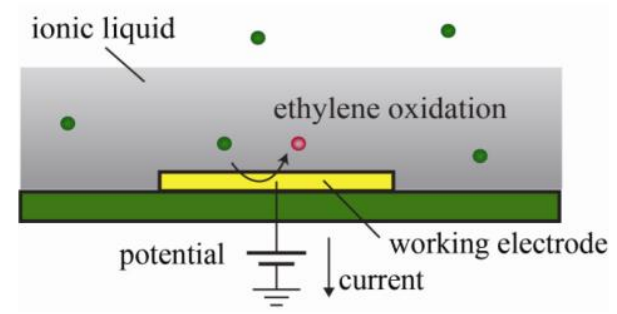

Fig. 1. Schematic representation of the ethylene detection mechanism, involving dissolution of ethylene in the ionic liquid and oxidation at the working electrode. 


\section{Sensor Fabrication}

The ethylene sensor was fabricated in four steps. First, $10 \mathrm{~nm} \mathrm{Ti}$ as adhesion layer and $100 \mathrm{~nm} \mathrm{Pt}$ were sputtered on a glass wafer. These were then patterned by photolithography and ion beam etching. This way, a disc-shaped $\mathrm{Pt}$ working electrode (WE) surrounded by a reference electrode (REF) was defined, as well as a rectangular counter electrode (CE) (see Fig. 2a). The feed wires and bond pads were also patterned in this step. Second, $200 \mathrm{~nm}$ $\mathrm{SiN}, 600 \mathrm{~nm} \mathrm{SiO} 2$ and $200 \mathrm{~nm} \mathrm{SiN} \mathrm{(NON} \mathrm{layer)}$ were deposited by plasma enhanced chemical vapor deposition as a capping layer preventing leakage from the feed wires. Third, holes were etched through the capping layer to re-expose WE, REF and CE. Finally, $10 \mathrm{~nm}$ Ti and $200 \mathrm{~nm}$ $\mathrm{Au}$ were deposited in the well of the WE by photolithography and lift-off. Fig. 2a shows a sensor die after fabrication. Dies were mounted and wirebonded in a dual in-line (DIL) package. To confine the IL on the electrode area, a well was created using a UV-curable epoxy, which also served to protect the bond wires (see Fig. $2 b)$.

a

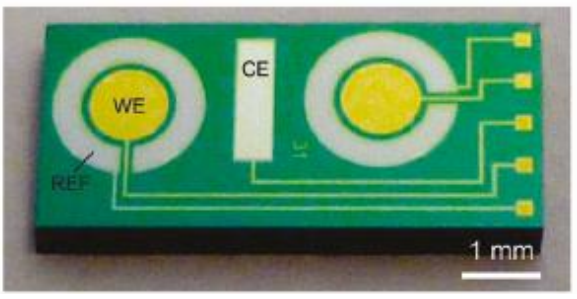

b

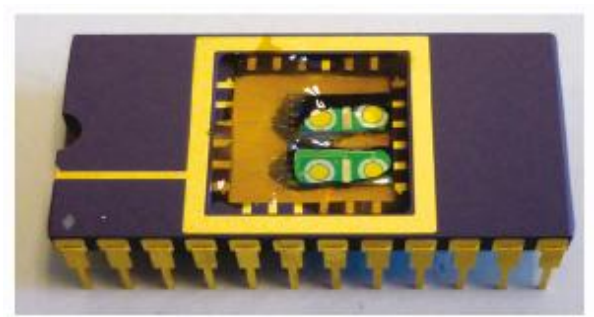

Fig. 2. a) Photograph of a fabricated ethylene sensor die (first generation). WE, REF and CE are indicated. Note that CE is shared between two sensors; b) Photograph of wirebonded devices.

The ionic liquids 1-butyl-3-methylimidazolium bis(trifluoromethylsulfonyl)imide [BMIM] $\left[\mathrm{NTf}_{2}\right]$ and 1-hexyl-3-methylimidazolium tris(pentafluoroethyl)trifluorophosphate ([HMIM][FAP] were obtained from Merck. Both were chosen because of their ethylene sorption properties. Before casting the IL, the working electrode was cleaned using piranha solution and extensive rinsing with deionized water. The IL film was formed by casting various amounts on the sensor surface using a $0.1-2.5 \mu \mathrm{L}$ precision pipette. Depending on the amount of IL deposited, the resulting IL film thickness was between 30 and $140 \mu \mathrm{m}$.

\section{Electrochemical Detection of Ethylene}

The device was mounted in a custom built gas flow cell and could be exposed to various ethylene concentrations in a nitrogen carrier flow. Part of the carrier flow was directed through water bubblers and mixed with dry nitrogen to control the humidity. Ethylene was added to the humidified carrier flow from a cylinder. All flow rates were controlled using mass flow controllers. All measurements were done at room temperature.

CVs recorded in the absence of ethylene showed the characteristic features associated with oxidation of the gold WE (peak current at approximatlely $1.1 \mathrm{~V}$ in the forward scan) and the subsequent reduction of the formed gold oxide (peak current at approximately $0.5 \mathrm{~V}$ in the backward scan) [5]. In the presence of ethylene, an extra peak appeared around $0.7 \mathrm{~V}$, before the onset of gold oxidation, attributed to the oxidation of ethylene (see Fig. 3).

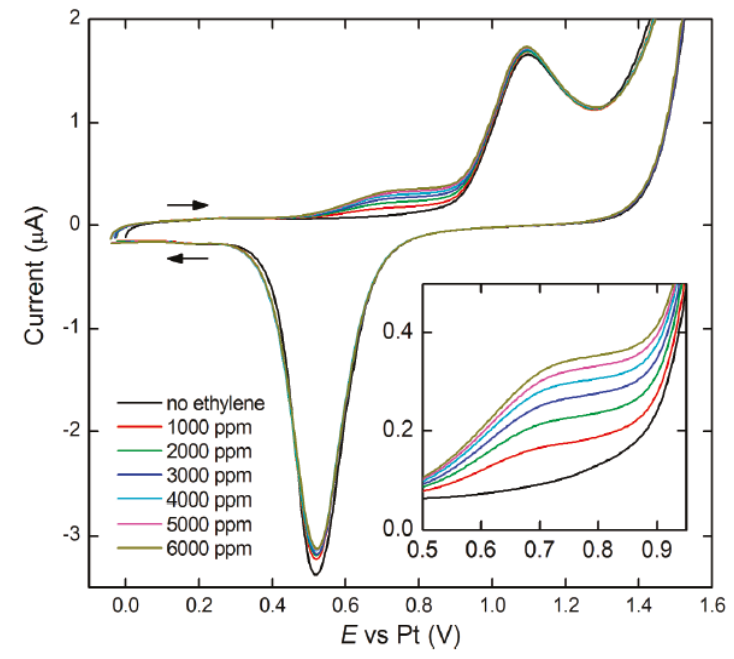

Fig. 3. Cyclic voltammograms recorded in the absence of ethylene (black line) and upon exposure to varying ethylene concentrations (colored lines). The inset shows a magnification of the ethylene oxidation window.

Real-time sensitive determination of the ethylene concentration was done by amperometric measurements in which the potential was set to a fixed value in the ethylene oxidation window and the current was recorded as a function of time. Under conditions where diffusion of ethylene to the electrode is the ratelimiting step, the steady-state current $I$ is then given by:

$l \propto \frac{A D c}{h}$ 
where $A$ is the working electrode area, $D$ is the diffusion coefficient of ethylene in the IL, $c$ is the concentration of ethylene in the IL and $h$ is the IL film thickness.

The steady state current was measured for various ethylene concentrations, humidity levels and IL film thicknesses [3]. In agreement with eq. (1), the current was found to increase with increasing ethylene concentration, and decrease with increasing IL film thickness. It was also found that the relative humidity has a large influence on the current, as the ethylene response increased with increasing humidity. This can be explained by the increased water content of the IL film, which decreases the viscosity and facilitates diffusion of ethylene. Thus, the humidity affects $D$ in eq (1).

To explore the detection limit of the sensor, it was operated under optimum conditions, i.e. using a thin IL film $(\sim 30 \mu \mathrm{m})$ and a high relative humidity ( 60\%). Figure 4 shows the current upon exposure to increasing concentrations of ethylene between 0 and $10 \mathrm{ppm}$. It is clearly seen that the presence of ethylene leads to an increased current, which is proportional to the ethylene concentration, as predicted by eq. (1). The response time is only a few seconds, and the detection limit (defined as three times the noise level) is approximately $750 \mathrm{ppb}$.

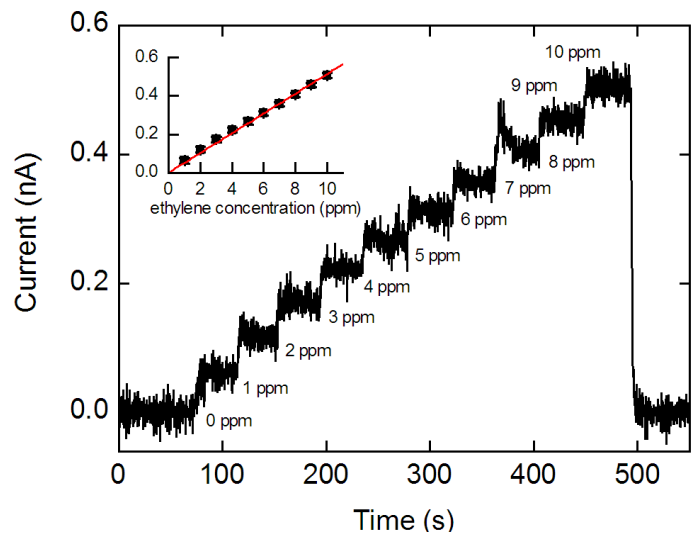

Fig. 4. Sensor response to an increasing ethylene concentration from 0 to $10 \mathrm{ppm}$. The inset shows the steady state current as a function of ethylene concentration and shows the linearity of the response.

\section{Sensor on Foil: Towards Smart Packaging}

Generally, electrochemical sensors have a closed reservoir containing the aqueous electrolyte. By using an IL electrolyte, which does not evaporate, no such reservoir is needed, thus allowing the planar and open device geometry employed here. Moreover, the sensor can not only be fabricated on rigid substrates such as $\mathrm{Si}$ or glass, but also on flexible materials, such as polymer foils. The use of polymer substrates can further reduce the fabrication costs of the devices. Fabricating the sensor on a flexible substrate also facilitates integration of the sensor in food packaging materials. Ultimately, this could lead to monitoring of the ripeness and quality of fruits and vegetables in individual packages. Adding sensing functionality to packaging materials is one of several strategies which are collectively referred to as smart or intelligent packaging $[6,7]$.

To demonstrate the feasibility of this approach, the ethylene sensor was fabricated on a $25 \mu \mathrm{m}$ thick polyethylene naphtalate (PEN) foil. The foil was laminated on a Si carrier wafer and planarized using a photoresist. For this second generation of sensors, an improved design with interdigitated WE and REF was chosen to minimize the distance between the WE and REF, preventing ohmic drop. Fig. 5a shows the design of the second generation sensor. Also, the fabrication process was simplified. First, 10 $\mathrm{nm} \mathrm{Ti}$ as adhesion layer and $100 \mathrm{~nm} \mathrm{Au}$ were sputtered on the substrate. These were then patterned by photolithography and lift-off. This way, an interdigitated Au working electrode (WE) was defined, surrounded by a square counter electrode (CE) (see Fig. 5a). The feed wires and bond pads were also patterned in this step. Then, $10 \mathrm{~nm} \mathrm{Ti}$ and $200 \mathrm{~nm} \mathrm{Pt}$ (REF) were deposited and patterned as an interdigitated electrode in between the fingers of the WE. The fabricated sensor is shown in Fig. $5 b$.

a
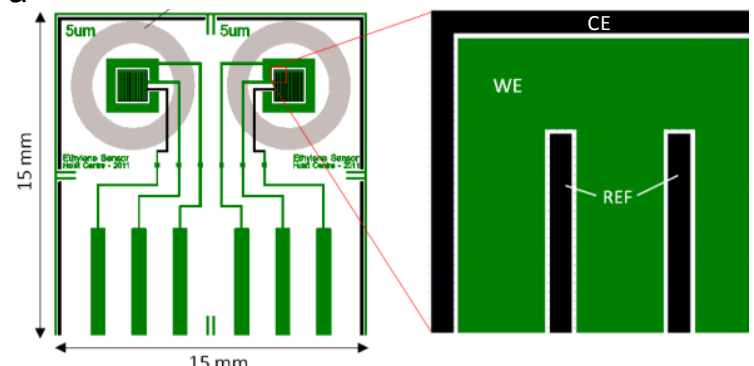

b

C

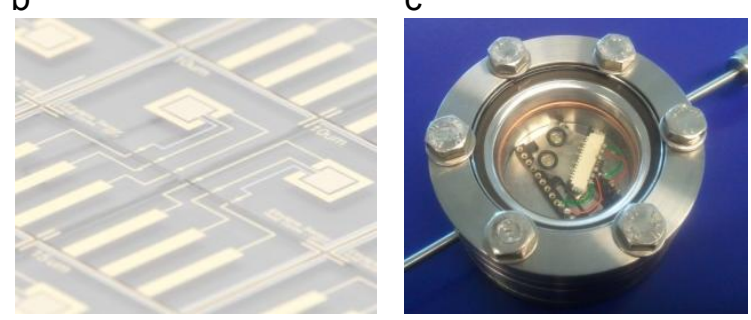

Fig. 5. a) Sensor design (second generation) with interdigitated WE and REF, surrounded by the square CE. The position of the O-ring is indicated by the gray circle; b) photograph of fabricated sensors; c) ethylene sensor on foil, mounted in the gas flow cell. 
Laser cutting was used to separate the dies before delaminating the foil from the carrier wafer. Instead of wirebonding in a DIL package, a ZIF connector was used for contacting the electrodes. Instead of the epoxy, a Viton O-ring, was glued to the surface to confine the IL. The completed sensor, mounted in the gas flow cell, is shown in Fig. 5c. A sensor die on foil measures $15 \times 15 \mathrm{~mm}^{2}$ and contains two sensors (see also the design shown in Fig. 5a).

The response of the foil-based sensor was tested by exposing it to $1000 \mathrm{ppm}$ ethylene. The result is shown in Fig. 6 and clearly demonstrates that a foil-based electrochemical ethylene sensor using an IL electrolyte was successfully fabricated.

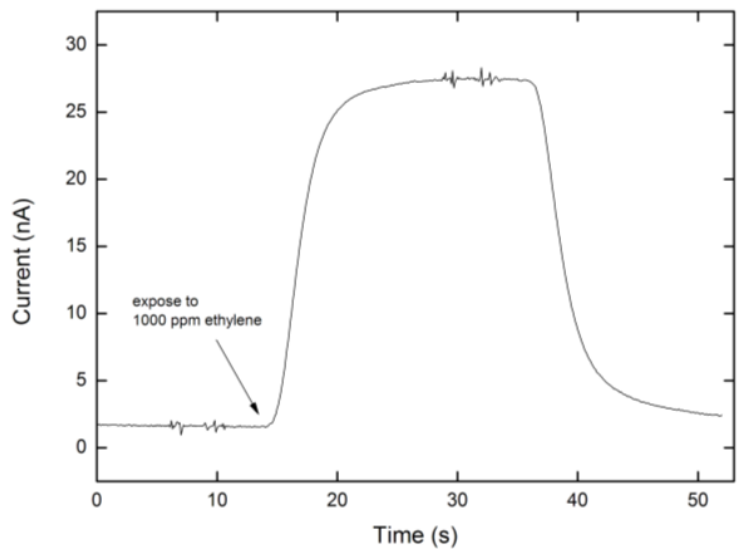

Fig. 6. Response of the foil-based sensor to 1000 ppm ethylene.

\section{Outlook}

With the current sensor design, detection of 1 ppm ethylene is possible. This is sufficient for certain applications, for example when ethylene is added to containers with fruits or vegetables to accelerate the ripening process, leading to relatively high ethylene concentrations. However, for monitoring the natural ripening process, a detection limit of well below $1 \mathrm{ppm}$ is desirable. For other applications, such as the monitoring of plant growth in greenhouses, even lower detection limits (down to single ppb level) are needed. Therefore, further improvement of the sensor, i.e. increasing the generated current, is being investigated.

By looking at eq. (1), several ways for increasing the current can be distinguished. First of all, the choice of IL is critical. The ideal IL would have a high ethylene solubility and a low Henry's constant (leading to high ethylene concentrations in the IL) and a low viscosity (leading to a high diffusion coefficient of ethylene in the IL). Secondly, the thickness of the IL film, which is currently tens of microns, could be reduced. Instead of depositing the pure IL on the substrate, it could be incorporated in a porous matrix or a polymer gel. Beside screening of different ILs, the addition of surfactants or other additives could further enhance the IL properties. Finally, increasing the electrode surface area and optimizing the electrode design could lead to a further increase of the current and improvement of sensor performance.

\section{Conclusions}

The use of IL electrolytes holds great promise for electrochemical gas sensors in general, and for ethylene detection in particular. Our results demonstrate that ionic liquids enable the fabrication of small, low cost and low power ethylene sensors with single ppm sensitivity. Thus, IL-based electrochemical ethylene sensors are promising candidates for monitoring and controlling the ripening of fruits and vegetables during transport and storage.

Furthermore, we have demonstrated the feasibility of fabricating our ethylene sensor on a flexible substrate. This is not only beneficial for reducing fabrication costs, it also opens up possibilities to integrate ethylene sensors in smart packaging materials.

\section{Acknowledgements}

The authors thank D. Wouters, V.A.T. Dam, J. Gonzalo-Ruiz, C. de Nooijer, Y. van Andel and $P$. Krusemann for their contribution to this work.

\section{References}

[1] R. Wills, B. McGlasson, D. Graham and D. Joyce Postharvest-An Introduction to the Physiology \& Handling of Fruit, Vegetables \& Ornamentals, 4th ed. UNSW Press: Sydney, Australia, 1998

[2] H. Dahms and J.O. Bockris Journal of the Electrochemical Society 111, 728-736 (1964); doi: 10.1149/1.2426221

[3] M.A.G. Zevenbergen, D. Wouters, V.A.T. Dam, S.H. Brongersma and M. Crego-Calama Analytical Chemistry 83, 6300-6307 (2011); doi: 10.1021/ac2009756

[4] D.S. Silvester Analyst 136, 4871-4882 (2011); doi: 10.1039/c1an15699c

[5] C. Zhao, G. Burrell, A.A.J. Torriero, F. Separovic, N.F. Dunlop, D.R. MacFarlane and A.M. Bond Journal of Physical Chemistry B 112 6923-6936 (2008); doi: 10.1021/jp711804j

[6] K.L. Yam, P.L. Takhistov and J. Miltz Journal of Food Science 70, R1-R10 (2004); doi: 10.1111/j.1365-2621.2005.tb09052.x

[7] A.L. Brody, B. Bugusu, J.H. Han, C. Koelsch and T.H. McHugh Journal of Food Science 73 R107R116 (2008); doi: 10.1111/j.1750-3841.2008.00933.x 